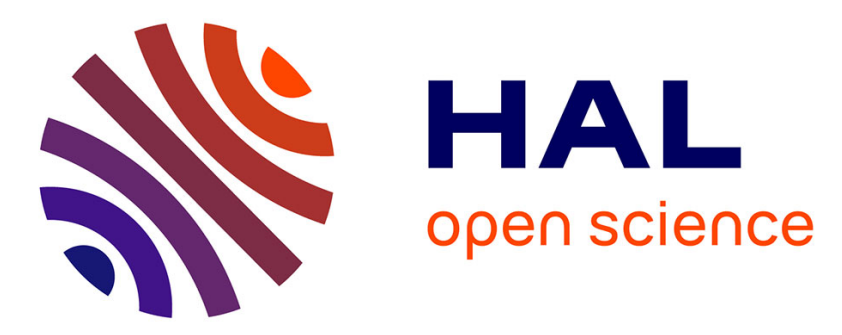

\title{
Physical performance and subjective ratings after a soccer-specific exercise simulation: Comparison of natural grass versus artificial turf
}

Mathieu Nedelec, Alan Mccall, Chris Carling, Franck Le Gall, Serge Berthoin, Grégory Dupont

\section{To cite this version:}

Mathieu Nedelec, Alan Mccall, Chris Carling, Franck Le Gall, Serge Berthoin, et al.. Physical performance and subjective ratings after a soccer-specific exercise simulation: Comparison of natural grass versus artificial turf. Journal of Sports Sciences, 2012, pp.1-8. 10.1080/02640414.2012.738923 . hal-01660898

\section{HAL Id: hal-01660898 \\ https: / hal-insep.archives-ouvertes.fr/hal-01660898}

Submitted on 11 Dec 2017

HAL is a multi-disciplinary open access archive for the deposit and dissemination of scientific research documents, whether they are published or not. The documents may come from teaching and research institutions in France or abroad, or from public or private research centers.
L'archive ouverte pluridisciplinaire HAL, est destinée au dépôt et à la diffusion de documents scientifiques de niveau recherche, publiés ou non, émanant des établissements d'enseignement et de recherche français ou étrangers, des laboratoires publics ou privés. 
This article was downloaded by: [FSSEP - Lille 2]

On: 07 November 2012, At: 07:17

Publisher: Routledge

Informa Ltd Registered in England and Wales Registered Number: 1072954 Registered office: Mortimer House, 37-41 Mortimer Street, London W1T 3J H, UK

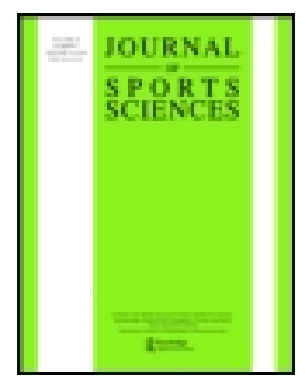

\title{
J ournal of Sports Sciences
}

Publication details, including instructions for authors and subscription information: http:// www.tandfonline.com/loi/ rjsp20

\section{Physical performance and subjective ratings after a soccer-specific exercise simulation: Comparison of natural grass versus artificial turf}

\author{
Mathieu Nédélec ${ }^{a b}$, Alan McCall ${ }^{b}$, Chris Carling ${ }^{b}$, Franck Le Gall ${ }^{b}$, Serge Berthoin ${ }^{a} \&$ \\ Grégory Dupont ${ }^{a} b$ \\ a University of Lille Nord de France, UDSL, EA 4488, 9 rue de I'Université, Ronchin, 59790, \\ France \\ ${ }^{b}$ LOSC Lille Métropole Football Club, LOSC Lab, Domaine de Luchin, Grand Rue, BP 79, \\ Camphin en Pevele, 59780, France \\ Version of record first published: 05 Nov 2012.
}

To cite this article: Mathieu Nédélec, Alan McCall, Chris Carling, Franck Le Gall, Serge Berthoin \& Grégory Dupont (2012): Physical performance and subjective ratings after a soccer-specific exercise simulation: Comparison of natural grass versus artificial turf, J ournal of Sports Sciences, DOI:10.1080/02640414.2012.738923

To link to this article: http:// dx. doi.org/ 10.1080/02640414.2012.738923

\section{irirst}

\section{PLEASE SCROLL DOWN FOR ARTICLE}

Full terms and conditions of use: http://www.tandfonline.com/page/terms-and-conditions

This article may be used for research, teaching, and private study purposes. Any substantial or systematic reproduction, redistribution, reselling, loan, sub-licensing, systematic supply, or distribution in any form to anyone is expressly forbidden.

The publisher does not give any warranty express or implied or make any representation that the contents will be complete or accurate or up to date. The accuracy of any instructions, formulae, and drug doses should be independently verified with primary sources. The publisher shall not be liable for any loss, actions, claims, proceedings, demand, or costs or damages whatsoever or howsoever caused arising directly or indirectly in connection with or arising out of the use of this material. 


\title{
Physical performance and subjective ratings after a soccer-specific exercise simulation: Comparison of natural grass versus artificial turf
}

\author{
MATHIEU NÉDÉLEC ${ }^{1,2}$, ALAN MCCALL ${ }^{2}$, CHRIS CARLING $^{2}$, FRANCK LE GALL ${ }^{2}$, \\ SERGE BERTHOIN ${ }^{1}$, \& GRÉGORY DUPONT ${ }^{1,2}$ \\ ${ }^{1}$ University of Lille Nord de France, France, UDSL, EA 4488, 9 rue de l'Université, Ronchin, 59790 France, and ${ }^{2}$ LOSC \\ Lille Métropole Football Club, LOSC Lab, Domaine de Luchin, Grand Rue, BP 79, Camphin en Pevele, 59780 France
}

(Accepted 5 October 2012)

\begin{abstract}
This study aimed to compare the recovery kinetics of physical performance and subjective ratings in response to a soccerspecific exercise simulation on natural grass and artificial turf. Physical performance tests and subjective ratings were assessed on 13 professional soccer players before, immediately after, $24 \mathrm{~h}$ and $48 \mathrm{~h}$ after the test. Physical performance tests included squat jump, countermovement jump, 6-s sprint on a non-motorised treadmill and isokinetic eccentric hamstring assessment $\left(2.09 \mathrm{rad} \cdot \mathrm{s}^{-1}\right)$. Hamstring peak torque decrement was higher $(P<0.05)$ on natural grass than on artificial turf immediately $(-4.0 \%$, CI $95 \%:-10.0$ to $2.0 \%$, Effect Size $[\mathrm{ES}]=0.29), 24 \mathrm{~h}(-3.1 \%$, CI $95 \%:-9.3$ to $3.1 \%$, ES =0.29) and $48 \mathrm{~h}(-3.8 \%$, CI $95 \%:-8.5$ to $0.9 \%, \mathrm{ES}=0.43)$ after the test. Squat jump performance decrement was significantly lower $(P<0.05)$ on natural grass than artificial turf $48 \mathrm{~h}$ after the test $(+3.7 \%$, CI $95 \%: 1.1$ to $6.3 \%, \mathrm{ES}=0.40) . \mathrm{Sprint}$ performance showed no change from baseline performance for both trials throughout the protocol. No significant interaction between surface and time was found for countermovement jump and subjective ratings. These results suggest that a one-off exercise on artificial turf does not induce greater fatigue nor does it delay the recovery process when compared to natural grass among regular artificial turf players.
\end{abstract}

Keywords: fatigue, recovery, football, field test, muscle soreness

\section{Introduction}

The International Football Association Board decided to include artificial turf pitches in the Laws of the Game in 2004. These surfaces are currently used for competitive league games at professional levels in several countries (e.g. France, Russia, and Switzerland) and for training purposes in many professional clubs. Professional players reported subjectively a greater physical effort during matches played on artificial turf than natural grass despite similar activity profiles (i.e. total distance covered, high-intensity running, number of sprints) and technical standard (i.e. standing tackles, headers) (Andersson, Ekblom, \& Krustrup, 2008a). Sassi et al. (2011) found a similar metabolic cost of running for both natural grass and artificial turf suggesting that such negative perceptions are not because of a higher cost of running, but due to other mechanical characteristics. In addition, Gains, Swedenhjelm, Mayhew, Bird, and Houser (2010) reported that change-in-direction speed during a one-off sprint is faster on artificial turf than on natural grass. This time differential between surfaces may be explained by more force being exerted during the change-in-direction motion resulting in more intense loading from accelerations and decelerations on artificial turf. Changes in direction, accelerations and decelerations are repetitively performed throughout a soccer match and induce muscle damage (Howatson \& Milak, 2009; Magalhães et al., 2010; Thompson, Nicholas, \& Williams, 1999). Young, Hepner, and Robbins (2012) found that players experiencing greater muscle damage $24 \mathrm{~h}$ post match covered significantly $(P<0.05)$ greater high-intensity running, accelerations and decelerations during the match.

The aim of the present study was to investigate the influence of playing surface on fatigue induced by changes in direction, accelerations and decelerations performed throughout a soccer match. The recovery kinetics of physical performance and subjective 
ratings in response to a standardised soccer-specific exercise simulation performed on natural grass and artificial turf were compared. A standardised soccerspecific exercise was used in order to control for the high variability of physical performance during a soccer match (Di Salvo, Gregson, Atkinson, Tordoff, \& Drust, 2009; Dupont et al., 2010). Based on previous findings (Gains et al., 2010), we hypothesised that post-exercise fatigue will be greater following the test on artificial turf resulting in delayed recovery process.

\section{Methods}

\section{Participants}

The participants were 13 professional soccer players (age: $17.7 \pm 0.5$ years; height: $180.2 \pm 6.0 \mathrm{~cm}$; body mass: $71.9 \pm 6.9 \mathrm{~kg}$; body fat: $9.4 \pm 2.0 \%$ ), but 12 were retained in the study, as one did not follow the recommendations. The players participated in one match and seven training sessions per week (volume: 11 to $14 \mathrm{~h}$ ). They were used to training and playing on both surfaces (natural grass and artificial turf) for at least 2 years.

\section{Experimental design}

The study involved a randomised crossover experimental design. On two separate occasions (natural grass vs. artificial turf), players completed three sessions separated by 2 or 3 weeks. Before the experimentation, players completed a medical examination. All players were fully informed of the purpose, benefits and risks involved with participation before giving their written informed consent. This investigation was led in accordance with the local Ethics Committee in Biomedical Research and the recommendation of the Helsinki Declaration.

\section{Experimental procedures}

Players were accustomed to rating the global intensity of training sessions using the modified Borg scale from 0 to 10 points (Borg, 1982; Foster, 1998) and the feeling scale from -5 to 5 points (Rejeski, Best, Griffith, \& Kenney, 1987). They were also familiar with rating their quality of sleep, fatigue, muscle soreness and stress using a scale from 1 to 7 points (Hooper, Mackinnon, Howard, Gordon, \& Bachmann, 1995) and to performing the following tests of physical performance: squat jump, countermovement jump, sprints on non-motorised treadmill, and isokinetic eccentric hamstring assessment. Two preliminary sessions were performed in order to verify the inter-day reliability of the physical performance tests and to collect reference values. During the first session in each condition, the 7-point Hooper's scale (Hooper et al., 1995), the total quality recovery perceived scale from 6 to 20 points (Kenttä \& Hassmén, 1998) and location of muscle soreness (Thompson et al., 1999) were collected before completing a $90 \mathrm{~min}$ soccer-specific aerobic field test (SAFT90; Small, McNaughton, Greig, \& Lovell, 2010). After completion of the $90 \mathrm{~min}$ soccer-specific aerobic field test and a 10 min rest interval, location of muscle soreness was recorded and physical performance tests performed in a testing room $200 \mathrm{~m}$ from the pitch. The second and third sessions corresponded to the subjective ratings, location of muscle soreness and physical performance tests performed $24 \mathrm{~h}$ and $48 \mathrm{~h}$ after the 90 min soccer-specific aerobic field test, respectively. Professional groundskeepers adjusted the soccer field watering to maintain the same experimental conditions. The $90 \mathrm{~min}$ soccer-specific aerobic field test on artificial turf was performed on third-generation artificial turf. The artificial turf pitch was not watered. The temperature ranged between $[10$ and $13^{\circ} \mathrm{C}$. Standardised verbal encouragement was provided during all the physical performance tests by experimenters. In order to limit dietary influences on test results, players were asked to follow standardised nutritional guidelines (quantity and content for food and drink) after each session and for breakfast, lunch and dinner. Each meal was eaten in the training centre. Participants were given written instructions to have their last meal at least $3 \mathrm{~h}$ before all testing sessions, and to avoid alcohol, tobacco and caffeine during the whole experimental period. During the period devoted to each condition, no training session was implemented and participants were requested not to use any different recovery treatments (cold bath, massage, compression garments), which may have affected the recovery pattern.

Players completed the $90 \mathrm{~min}$ soccer-specific aerobic field test protocol, which consists of two $45 \mathrm{~min}$ periods interceded by a $15 \mathrm{~min}$ passive rest period (half-time), performed as a shuttle run test over a $20 \mathrm{~m}$ distance (Small et al., 2010). The $90 \mathrm{~min}$ soccer-specific aerobic field test is designed to replicate the fatigue responses to soccer matchplay and includes multiple backwards running, sidestepping, changes in direction and frequent acceleration and deceleration actions inherent to match-play. Thirty-six maximal shooting actions were performed during the $90 \mathrm{~min}$ soccer-specific aerobic field test protocol to increase the load to the quadriceps reflective of match-play (Small et al., 2010). Prior to the $90 \mathrm{~min}$ soccer-specific aerobic field test, players participated in a standardised warm-up performed on the surface on which they had to complete the test. The warm-up was the same 
as that used before a match and included $10 \mathrm{~min}$ light jogging $\left(9-11 \mathrm{~km} \cdot \mathrm{h}^{-1}\right)$, dynamic activities (buttock kicks, high knee lifts, backwards running, sidestepping), sprints and familiarisation with the $90 \mathrm{~min}$ soccer-specific aerobic field test exercise protocol for a total duration of $15 \mathrm{~min}$. Before the experimentation, players were asked to choose soccer boots that they would be required to wear in both conditions (natural grass and artificial turf). An experimenter checked that soccer boots worn by players were the same during each condition (natural grass and artificial turf). Players' nude body mass was recorded immediately before and after the $90 \mathrm{~min}$ soccer-specific aerobic field test with a digital scale (Seca 780, Hamburg, Germany). During half-time, players drank a sports drink containing 6\% carbohydrate (Gatorade, PepsiCo, United States). The hydration plan was the same as that used during a match with players free to choose the fluid intake to the upper limit of 11 . Players' fluid intake during the first condition was recorded and players consumed the same fluid intake during the second condition. The fluid loss was calculated by the following formula: Fluid loss $=$ (body mass post-test - body mass pre-test) + fluid intake (Andersson et al., 2008b).

The order of physical performance tests was identical throughout each protocol and included the following: squat jumps, countermovement jumps, 6-s sprints and isokinetic eccentric hamstring assessment. Players performed three squat jumps and three countermovement jumps on a force platform (Kistler AG, Winterhur, Switzerland) with built-in charge amplifier. The force signal was sampled at $1000 \mathrm{~Hz}$. A 1-min rest period was set between each jump. The best jump from three attempts was recorded. For the squat jump, participants were instructed to bend the knees at $90^{\circ}$, pausing for $3 \mathrm{~s}$ before jumping upwards on the verbal command 'go'. A goniometer (Lafayette Instrument Company, USA) was used to set the angle. For the countermovement jump, participants were instructed to jump explosively upwards immediately after descending to a self-selected depth. During both types of jump tests, the players placed their hands on their hips. The inter-day test-retest reliability for squat jump and countermovement jump was very high: the typical error (TE) was 1.4 and $1.5 \mathrm{~cm}$, respectively, the intra-class correlation coefficient (ICC) was 0.90 and 0.92 , respectively, while coefficient of variation (CV) was $3.1 \%$ and $2.9 \%$, respectively. Players completed three 6-s sprints separated by $3 \mathrm{~min}$ of passive recovery on a non-motorised treadmill (Woodway Force 3.0, Waukesha, USA). Start position (standing start with hand on the handles) was standardised. The best value from three sprints was recorded for mean power output, mean speed and peak speed. Very high inter-day test-retest reliability was found for mean power output, mean speed and peak speed (TE: $90 \mathrm{~W}, 0.2 \mathrm{~m} \cdot \mathrm{s}^{-1}$ and $0.2 \mathrm{~m} \cdot \mathrm{s}^{-1}$, respectively; ICC: $0.87,0.89$ and 0.88 , respectively; CV: $3.1 \%$, $2.6 \%$ and $2.2 \%$, respectively). The non-motorised treadmill was calibrated before each test. Treadmill belt speed, distance and horizontal forces were collected at a sampling rate of $100 \mathrm{~Hz}$ via the XPV7 PCB interface (Fitness Technology, Adelaide, Australia) and analysed with the Force 3.0 Software (Innervations Software, Joondalup, Australia). Players performed three successive maximal voluntary isokinetic eccentric hamstring actions without rest on a dynamometer (Con-Trex, Duebendorf, Switzerland). During testing, players were seated on the dynamometer in an adjustable chair, with test positions recorded and repeated for each player in subsequent sessions. Actions were performed on the players' dominant leg (their 'kicking' leg) through a range of $90^{\circ}$ (with $0^{\circ}$ being full knee extension) at an isokinetic angular velocity of $2.09 \mathrm{rad} \cdot \mathrm{s}^{-1}\left(120^{\circ} \mathrm{s}^{-1}\right)$. Hamstring peak torque was recorded. Peak torque showed very high interday test-retest reliability (TE: $7.6 \mathrm{~N} \cdot \mathrm{m}$; ICC: 0.87; CV: $4.7 \%)$.

Heart rate was continuously monitored throughout the $90 \mathrm{~min}$ soccer-specific aerobic field test (Polar Team System, Kempele, Finland) with heart rate values averaged every $5 \mathrm{~s}$.

At the beginning of each session, players were required to rate their quality of sleep, fatigue, muscle soreness and stress on the 7-point Hooper's scale (Hooper et al., 1995). They used highlighter to specify where they experienced muscle soreness (Thompson et al., 1999). Players were also asked to rate their recovery as an overall psycho-physiological rating for the previous 24 hours, including the previous night's sleep, using the total quality recovery perceived scale (Kenttä \& Hassmén, 1998). After the 90 min soccer-specific aerobic field test, participants were required to rate the global intensity of the session using the modified Borg scale (Borg, 1982; Foster, 1998) and the feeling scale (Rejeski et al., 1987). Ratings of fatigue, muscle soreness and stress levels as well as location of muscle soreness were also collected immediately after the $90 \mathrm{~min}$ soccer-specific aerobic field test. Baseline values corresponded to values obtained the morning before the $90 \mathrm{~min}$ soccer-specific aerobic field test.

\section{Statistical analysis}

Simple descriptive statistics are reported as means \pm standard deviations (mean $\pm s$ ). The normality distribution of the data was checked with the 
Shapiro-Wilk test. Comparison between conditions (natural grass vs. artificial turf) was analysed using 2way analysis of variance (ANOVA) for repeated measures. The effects of the independent variables (surface and time) on the dependent variables squat jump, countermovement jump, mean power output, mean speed, peak speed, hamstring peak torque and subjective ratings - were analysed using a 2-way ANOVA for repeated measures. Bonferroni post hoc was then applied when the significant Fvalue was found. Changes in the mean between reference and post-90 min soccer-specific aerobic field test testing values of the two conditions were expressed as a percentage of the reference values for objective tests and absolute values for subjective ratings. Comparisons between surfaces were assessed through the difference in change scores. Effect size data (ES) was calculated to determine the magnitude of the change score and was assessed using the following criteria: $<0.2=$ trivial, $0.2-0.6=$ small, $0.6-1.2=$ moderate, $\quad 1.2-2.0=$ large, and $>2.0=$ very large (Hopkins, 2002). Concerning the diagram labelling of the body's musculature, differences in frequencies in muscle areas highlighted as sore between the two conditions were tested using the following criteria: $<10 \%=$ trivial, $10-30 \%=$ small, $30-50 \%=$ moderate, $50-70 \%=$ large, $>70 \%=$ very large (Hopkins, 2002). Differences in heart rate, fluid loss, body mass and rating of the $90 \mathrm{~min}$ soccer-specific aerobic field test were tested for significance using the Student's paired t-test when parametric methods were used or the paired Wilcoxon test when non-parametric methods were used. Confidence intervals (CI 95\%) were used to specify estimation of changes in performance tests, subjective ratings and differences in frequencies. Statistical significance was set at $P<0.05$.

\section{Results}

90 min soccer-specific aerobic field test

No significant differences were observed between the mean heart rate during the $90 \mathrm{~min}$ soccer-specific aerobic field test on artificial turf $(151 \pm 15 \mathrm{bpm})$ and the mean heart rate during the test on natural grass $(145 \pm 14 \mathrm{bpm})$. Similarly, no significant difference was observed between the fluid loss during the $90 \mathrm{~min}$ soccer-specific aerobic field test on artificial turf $(1321 \pm 855 \mathrm{ml})$ and the fluid loss during the test on natural grass $(1554 \pm 480 \mathrm{ml})$. The body mass measured after the 90 min soccerspecific aerobic field test on both surfaces was significantly lower $(P<0.05)$ than those recorded before the test, with a loss of body mass of $-0.7 \pm 0.8 \mathrm{~kg}(-0.9 \pm 1.0 \%)$ on artificial turf and a loss of body mass of $-0.9 \pm 0.5 \mathrm{~kg}(-1.3 \pm 0.6 \%)$ on natural grass. The fluid intake in both conditions was $638 \pm 158 \mathrm{ml}$. No significant differences were found for the rating of intensity after the $90 \mathrm{~min}$ soccer-specific aerobic field test performed on artificial turf and natural grass $(4.3 \pm 1.5$ vs. $4.8 \pm 2.2$ respectively) or for the feeling scale $(1.0 \pm 2.4$ vs. $1.4 \pm 1.8$ respectively).

Recovery kinetics for physical performance and subjective ratings after the 90 min soccer-specific aerobic field test

The effect of surface on physical performance and subjective ratings and comparisons between surfaces throughout the recovery period are presented in Tables I and II. A significant interaction was found for squat jump between surface and time $(P<0.01)$. Post hoc analysis revealed that squat jump performance decrement was significantly lower $(P<0.05)$ on natural grass than artificial turf $48 \mathrm{~h}$ after the test with a small difference $(+3.7 \%$, CI $95 \%$ : 1.1 to $6.3 \%, \mathrm{ES}=0.40)$ observed. A significant main effect for time was also found for the squat jump $(P<0.001)$. Post hoc analysis revealed that squat jump performance was significantly impaired immediately after the test $(P<0.001)$. No significant interaction was found for countermovement jump between surface and time with only trivial differences $(\mathrm{ES}=0.04-0.12)$ between artificial turf and natural grass in changes in countermovement jump performance throughout the recovery period. However, a significant main effect for time was found for countermovement jump $(P<0.01)$. Post hoc analysis revealed that countermovement jump performance was significantly impaired immediately after the test $(P<0.01)$ and at $24 \mathrm{~h}(P<0.05)$.

There was no interaction effect of surface and time on hamstring peak torque. However, there was a main effect of surface on hamstring peak torque $(P<0.05)$. Hamstring peak torque decrement was higher on natural grass than on artificial turf with small differences immediately $(-4.0 \%$, CI $95 \%$ : -10.0 to $2.0 \%, \mathrm{ES}=0.29), 24 \mathrm{~h}(-3.1 \%, \mathrm{CI} 95 \%:-9.3$ to $3.1 \%, \mathrm{ES}=0.29)$ and $48 \mathrm{~h}(-3.8 \%, \mathrm{CI} 95 \%:-8.5$ to $0.9 \%, \mathrm{ES}=0.43)$ after the $90 \mathrm{~min}$ soccer-specific aerobic field test. There was also a main effect of time on hamstring peak torque $(P<0.05)$. Post hoc analysis revealed that hamstring peak torque was significantly different from baseline immediately after the test and at $24 \mathrm{~h}(P<0.05)$.

All three variables reflective of sprint performance (i.e. mean power output, mean speed and peak speed) showed no change from baseline performance for both trials throughout the protocol. There were only trivial differences ( $\mathrm{ES}=0.01-0.17)$ between artificial turf and natural grass on changes in mean power output, mean speed and peak speed. 

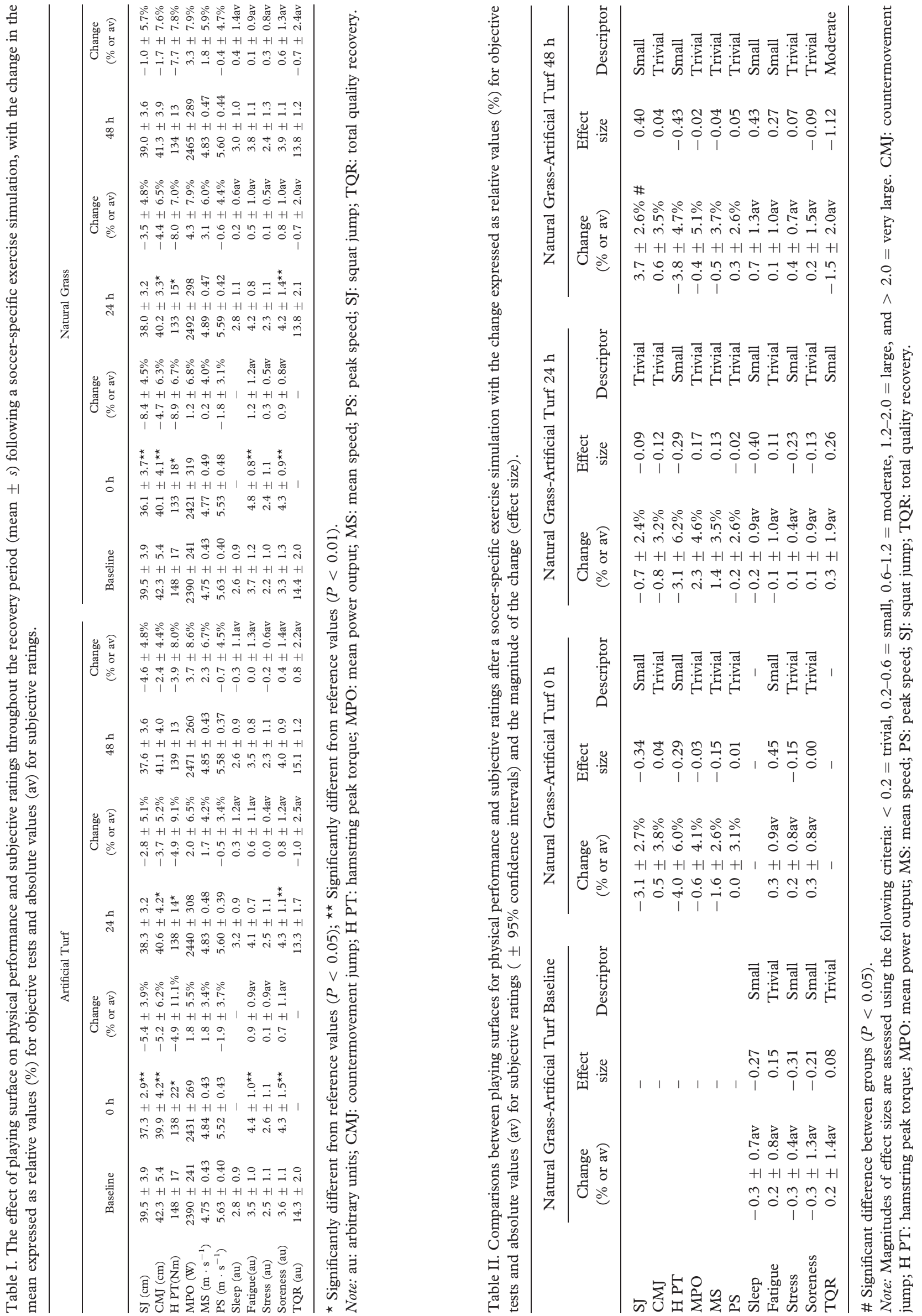
There was no interaction effect of surface and time on ratings of quality of sleep, fatigue, muscle soreness, stress and total quality recovery with only trivial to small differences $(\mathrm{ES}=0.00-0.45)$ between artificial turf and natural grass on changes in sleep, fatigue, stress and muscle soreness ratings throughout the recovery period. However, for the variable fatigue, there was a main effect of time $(P<0.001)$ with an increase to 'average-high' (1 unit) for both trials observed immediately after the test $(P<0.001)$. For the variable muscle soreness, a main effect of time was also observed $(P<0.01)$ with significant increases observed immediately after the test and at $24 \mathrm{~h}$ compared with baseline values $(P<0.01)$.

Differences in frequencies in muscle areas highlighted as sore between the two conditions at different time points throughout the recovery period are shown in Table III. There were trivial or small differences for pubis, groin, tibialis and lower back. However, soreness in quadriceps immediately after the $90 \mathrm{~min}$ soccer-specific aerobic field test, in gluteus $24 \mathrm{~h}$ after the test and in hamstring $48 \mathrm{~h}$ after the test were all reported to be moderately lower (from 31 to $46 \%$ ) on natural grass than artificial turf.

\section{Discussion}

The aim of the present study was to compare the recovery kinetics of physical performance and subjective ratings in response to a soccer-specific exercise test performed on natural grass and artificial turf. The 90 min soccer-specific aerobic field test is validated to replicate the movement demands of soccer match-play and includes multiple changes in direction, accelerations and decelerations associated with muscle damage (Howatson \& Milak, 2009; Magalhães et al., 2010; Thompson et al., 1999). Based on previous findings, we hypothesised that the soccer test-induced muscle damage may be greater on artificial turf resulting in delayed recovery process. Warren, Lowe, and Armstrong (1999) stated that measurement of maximal voluntary contraction torque provides the best method for quantifying muscle damage as it is accurate and reliable. In the present study, eccentric hamstring torque was tested because the hamstring is particularly prone to injury (Woods et al., 2004) and fatigue in soccer (Greig, 2008; Small et al., 2010). Results show that our hypothesis was rejected since hamstring peak torque decrement was higher on natural grass than on artificial turf $(P<0.05)$ with small differences reported through the $48 \mathrm{~h}$ recovery period. Yet despite the higher peak torque decrement on natural grass, players reported moderately higher soreness in the hamstrings in the artificial turf condition $48 \mathrm{~h}$ after the $90 \mathrm{~min}$ soccer-specific aerobic field test confirming that soreness is poorly correlated with changes in muscle function (Warren et al., 1999). Here, 6-s sprint performance (i.e. mean power output, mean speed) was not affected throughout the recovery period. This result may be explained by the activity profile of the 90 min soccer-

Table III. Frequencies difference ( $\pm 95 \%$ confidence intervals) in muscle areas highlighted as sore between the two conditions throughout the recovery period.

\begin{tabular}{|c|c|c|c|c|c|c|c|c|}
\hline & \multicolumn{2}{|c|}{ Baseline } & \multicolumn{2}{|c|}{$0 \mathrm{~h}$} & \multicolumn{2}{|c|}{$+24 \mathrm{~h}$} & \multicolumn{2}{|c|}{$+48 \mathrm{~h}$} \\
\hline & $\begin{array}{l}\text { Freq. } \\
\text { diff. }(\%)\end{array}$ & Descriptor & $\begin{array}{l}\text { Freq. } \\
\text { diff. }(\%)\end{array}$ & Descriptor & $\begin{array}{l}\text { Freq. } \\
\text { diff. }(\%)\end{array}$ & Descriptor & $\begin{array}{c}\text { Freq. } \\
\text { diff. (\%) }\end{array}$ & Descriptor \\
\hline \multicolumn{9}{|l|}{ Anterior view } \\
\hline Pubis & $-8 \pm 24$ & Trivial & $-15 \pm 27$ & Small & $-8 \pm 14$ & Trivial & $-8 \pm 14$ & Trivial \\
\hline Left groin & $23 \pm 29$ & Small & $-15 \pm 35$ & Small & $23 \pm 35$ & Small & $-8 \pm 30$ & Trivial \\
\hline Right groin & $15 \pm 27$ & Small & $-15 \pm 32$ & Small & $23 \pm 35$ & Small & $-15 \pm 32$ & Small \\
\hline Left quadriceps & $0 \pm 20$ & Trivial & $46 \pm 31$ & Moderate & $23 \pm 33$ & Small & $15 \pm 27$ & Small \\
\hline Right quadriceps & $0 \pm 20$ & Trivial & $46 \pm 31$ & Moderate & $15 \pm 35$ & Small & $8 \pm 30$ & Trivial \\
\hline Left tibialis & $0 \pm 0$ & Trivial & $0 \pm 0$ & Trivial & $0 \pm 0$ & Trivial & $0 \pm 0$ & Trivial \\
\hline $\begin{array}{l}\text { Right tibialis } \\
\text { Posterior view }\end{array}$ & $15 \pm 20$ & Small & $8 \pm 14$ & Trivial & $8 \pm 14$ & Trivial & $0 \pm 0$ & Trivial \\
\hline Lower back & $-15 \pm 35$ & Small & $-23 \pm 35$ & Small & $-15 \pm 37$ & Small & $-15 \pm 37$ & Small \\
\hline Left gluteus & $15 \pm 20$ & Small & $23 \pm 29$ & Small & $38 \pm 26$ & Moderate & $-15 \pm 27$ & Small \\
\hline Right gluteus & $8 \pm 14$ & Trivial & $23 \pm 29$ & Small & $31 \pm 25$ & Moderate & $-15 \pm 32$ & Small \\
\hline Left hamstring & $-8 \pm 36$ & Trivial & $-15 \pm 37$ & Small & $15 \pm 37$ & Small & $38 \pm 35$ & Moderate \\
\hline Right hamstring & $0 \pm 37$ & Trivial & $-23 \pm 35$ & Small & $23 \pm 29$ & Small & $46 \pm 27$ & Moderate \\
\hline Left calf & $0 \pm 20$ & Trivial & $0 \pm 32$ & Trivial & $-15 \pm 35$ & Small & $23 \pm 29$ & Small \\
\hline Right calf & $8 \pm 24$ & Trivial & $-8 \pm 34$ & Trivial & $-8 \pm 36$ & Trivial & $8 \pm 30$ & Trivial \\
\hline
\end{tabular}

Note: Magnitudes of effect sizes are assessed using the following criteria: $<10 \%=$ trivial, $10-30 \%=$ small, $30-50 \%=$ moderate, $50-$ $70 \%=$ large, $>70 \%=$ very large. For a given area, a positive value in a frequencies difference indicates that more players experienced soreness in the artificial turf condition than the natural grass condition, while a negative value in a frequencies difference indicates that more players experienced soreness in the natural grass condition than the artificial turf condition. 
specific aerobic field test which does not include contact situations such as tackles or collisions between players observed during actual soccer match-play. In a comparison of the effect of a simulated team sport activity circuit either with or without 44 body contacts on sprint performance, Singh, Guelfi, Landers, Dawson, and Bishop (2011) found that performance was significantly slower $48 \mathrm{~h}$ following the protocol with body contact $(P<0.05)$. In contrast, performance was maintained $48 \mathrm{~h}$ after the protocol without body contact. Similarly, Pointon and Duffield (2012) found that an intermittentsprint protocol with tackling resulted in a significantly slower mean sprint time compared to the same protocol without tackling $(P<0.05)$. This study proposed that the inclusion of tackling resulted in greater central fatigue compared to the control condition, as observed by a greater reduction in voluntary activation. The absence of any 6-s sprint performance impairment in the present study may therefore be attributed to insufficient levels of muscle damage resulting from the lack of contact actions, jumps and tackles. As a consequence, future studies investigating the recovery process after a soccer match simulation test should consider the inclusion of simulated contact, jumps and tackles, in the exercise protocol. Future studies may also compare the impact of a soccer match and the 90 min soccerspecific aerobic field test on muscle damage markers.

In the present study, no significant differences were observed between the mean heart rate during 90 min soccer-specific aerobic field test on artificial turf and natural grass which suggests a similar physiological load on both surfaces. The rating of the global intensity of the $90 \mathrm{~min}$ test performed on natural grass and artificial turf showed no significant difference as did the feeling during the test which was 'neutral-slightly good' on both surfaces. Andersson et al. (2008a) examined the movement patterns, ball skills, and the impressions of elite football players during competitive games on artificial turf and natural grass. On a 10 -point scale, where $0=$ 'better than' and $10=$ 'worse than', players reported a negative overall impression $(8.3 \pm 0.2)$, poorer ball control $(7.3 \pm 0.3)$, and greater subjective physical effort $(7.2 \pm 0.2)$ on artificial turf than natural grass despite similar total distance covered, high-intensity running and number of sprints. The discrepancy between our results and those from Andersson et al. (2008a) could be due to the protocol used and/or the familiarisation with artificial turf. In the present study, players completed a standardised soccer test which did not include any changes in playing characteristics during matches (i.e. fewer sliding tackles and more short passes on artificial turf) reported by Andersson et al. (2008a). The absence of a negative impression of artificial turf in the present study may also be explained by the fact that we tested young players (17.7 years) who were accustomed to playing on artificial turf whereas Andersson et al. (2008a) tested predominantly regular natural grass players aged 28.8 years. Familiarisation is a key point in studying the recovery process. Lavender and Nosaka (2008) have shown that a light eccentric exercise, which does not induce changes in any of the indirect markers of muscle damage, confers protection against muscle damage after a more strenuous eccentric exercise performed two days later. In the present study, the absence of negative perceptions may likely be explained by the familiarisation with artificial turf, but also the timing of the test (almost the end of the season). The familiarisation with artificial turf may consequently be important when measuring players' impression of artificial turf versus natural grass.

\section{Conclusion}

Findings from the present study indicate that although within-condition differences can be observed in physical performance and subjective ratings after a soccer test designed to replicate the physiological and mechanical demands of soccer match-play, there is no evidence to indicate that exercise on artificial turf results in greater fatigue and delayed recovery process. Future studies are required to confirm that results are similar when exercise is performed on a surface which players are not accustomed to since non-regular artificial turf players anecdotally report that the acute transition from natural grass to artificial turf is particularly disturbing.

\section{References}

Andersson, H., Ekblom, B., \& Krustrup, P. (2008a). Elite football on artificial turf versus natural grass: Movement patterns, technical standards, and player impressions. Fournal of Sports Sciences, 26, 113-122.

Andersson, H., Raastad, T., Nilsson, J., Paulsen, G., Garthe, I., \& Kadi, F. (2008b). Neuromuscular fatigue and recovery in elite female soccer: Effects of active recovery. Medicine and Science in Sports and Exercise, 40, 372-380.

Borg, G.A. (1982). Psychophysical bases of perceived exertion. Medicine and Science in Sports and Exercise, 14, 377-381.

Di Salvo, V., Gregson, W., Atkinson, G., Tordoff, P., \& Drust, B. (2009). Analysis of high intensity activity in Premier League soccer. International fournal of Sports Medicine, 30, 205-212.

Dupont, G., Nedelec, M., McCall, A., McCormack, D., Berthoin, S., \& Wisløff, U. (2010). Effect of 2 soccer matches in a week on physical performance and injury rate. The American fournal of Sports Medicine, 38, 1752-1758.

Foster, C. (1998). Monitoring training in athletes with reference to overtraining syndrome. Medicine and Science in Sports and Exercise, 30, 1164-1168.

Gains, G.L., Swedenhjelm, A.N., Mayhew, J.L., Bird, H.M., \& Houser, J.J. (2010). Comparison of speed and agility performance of college football players on field turf and natural grass. fournal of Strength and Conditioning Research, 24, 2613-2617. 
Greig, M. (2008). The influence of soccer-specific fatigue on peak isokinetic torque production of the knee flexors and extensors. The American fournal of Sports Medicine, 36, 1403-1409.

Hooper, S.L., Mackinnon, L.T., Howard, A., Gordon, R.D., \& Bachmann, A.W. (1995). Markers for monitoring overtraining and recovery. Medicine and Science in Sports and Exercise, 27, 106-112.

Hopkins, W.G. (2002). A scale of magnitudes for effect statistics. Sportscience. Retrieved from http://sportsci.org/resource/stats/ index.html

Howatson, G., \& Milak, A. (2009). Exercise-induced muscle damage following a bout of sport specific repeated sprints. Fournal of Strength and Conditioning Research, 23, 2419-2424.

Kenttä, G., \& Hassmén, P. (1998). Overtraining and recovery. $A$ conceptual model. Sports Medicine, 26, 1-16.

Lavender, A.P., \& Nosaka, K. (2008). A light load eccentric exercise confers protection against a subsequent bout of more demanding eccentric exercise. Fournal of Science and Medicine in Sport, 11, 291-298.

Magalhães, J., Rebelo, A., Oliveira, E., Silva, J.R., Marques, F., \& Ascensão, A. (2010). Impact of Loughborough Intermittent Shuttle Test versus soccer match on physiological, biochemical and neuromuscular parameters. European Fournal of Applied Physiology, 108, 39-48.

Pointon, M., \& Duffield, R. (2012). Cold water immersion recovery after simulated collision sport exercise. Medicine and Science in Sports and Exercise, 44, 206-216.

Rejeski, W.J., Best, L.D., Griffith, P., \& Kenney, E. (1987). Sexrole orientation and the responses of men to exercise stress. Research Quarterly for Exercise and Sport, 58, 260-264.
Sassi, A., Stefanescu, A., Menaspa', P., Bosio, A., Riggio, M., \& Rampinini, E. (2011). The cost of running on natural grass and artificial turf surfaces. Fournal of Strength and Conditioning Research, 25, 606-611.

Singh, T.K., Guelfi, K.J., Landers, G., Dawson, B., \& Bishop, D. (2011). A comparison of muscle damage, soreness and performance following a simulated contact and non-contact team sport activity circuit. Fournal of Science and Medicine in Sport, 14, 441-446.

Small, K., McNaughton, L., Greig, M., \& Lovell, R. (2010). The effects of multidirectional soccer-specific fatigue on markers of hamstring injury risk. Fournal of Science and Medicine in Sport, 13, 120-125.

Thompson, D., Nicholas, C.W., \& Williams, C. (1999). Muscular soreness following prolonged intermittent high-intensity shuttle running. Fournal of Sports Sciences, 17, 387-395.

Warren, G.L., Lowe, D.A., \& Armstrong, R.B. (1999). Measurement tools used in the study of eccentric contraction-induced injury. Sports Medicine, 27, 43-59.

Woods, C., Hawkins, R.D., Maltby, S., Hulse, M., Thomas, A., \& Hodson, A. (2004). The Football Association Medical Research Programme: An audit of injuries in professional footballanalysis of hamstring injuries. British fournal of Sports Medicine, 38, 36-41.

Young, W.B., Hepner, J., \& Robbins, D.W. (2012). Movement demands in Australian Rules football as indicators of muscle damage. Fournal of Strength and Conditioning Research, 26, 492496. 